\title{
Mycotic Aneurysm of Aorta Following Mitral Valve Replacement - A Case Report
}

\author{
AMA Rahim ${ }^{1}$, MH Zaman ${ }^{1}$, GMM Hossain ${ }^{2}$, SAN Alam ${ }^{2}$, MA Quashem ${ }^{1}$, NU Ahmed ${ }^{1}$, KN Mahmood $^{1}$ \\ ${ }^{1}$ Department of Cardiac Surgery, NICVD, Dhaka. ${ }^{2}$ Department of Vascular Surgery, NICVD, Dhaka.
}

\begin{abstract}
Key words:
Mycotic

aneurysm of

Aorta, Mitral

valve

Replacement.

The term mycotic aneurysm refers to aneurysm associated with infection by microorganism. Sir William Osler first coined the term mycotic aneurysm in 1885 by disclosing the relation between abnormal cardiac valves and infection with micrococci not with fungi. An 11 years old female from Feni presented with asymptomatic vascular swelling in abdomen referred by a cardiologist.

$C T$ angiogram revealed fusiform aneurysm in distal part of abdominal aorta involving ostioproximal part of both common iliac arteries and saccular aneurysm of distal part of superior mesenteric arteries suggestive of mycotic aneurysm. Patient underwent vascular operation aorto biilliac bypass by PTFE graft with excision and ligation of aneurysm of superior mesenteric arteries .Mycotic aneurysm in bacterial endocarditis is rare. It is a challenging job for the cardiologists, infectious disease specialists and vascular surgeon. Time appropriate skilled prompt surgical management can bring smile for both patients and physicians.
\end{abstract}

Abstract:

(Cardiovasc. j. 2012; 5(1): 107-111)

\section{Introduction:}

Aneurysm is defined as localized dilatation at least 1.5times the diameter of a normal artery. Abdominal aortic aneurysms are the $13^{\text {th }}$ leading cause of death, accounting more than 15,000 deaths per year. Sir William Osler first coined the term mycotic aneurysm in 1885 by disclosing the relation between abnormal cardiac valves and infection with micrococci not with fungi. Classic mycotic aneurysm now refers to narrow clinical spectrum of infection of arterial wall found in association with endocarditis. The term mycotic aneurysm is refer to aneurysm associated with infection of microorganism not fungi; although it appeared as infection extending to aorta like fresh fungus vegetations previously well known as so called mycotic endarteritis. Mycotic aneurysms in aorta are saccular (localized/multiple), asymmetric pseudo aneurysm reminiscent of a mushroom. Recently less than $10 \%$ cases of mycotic aneurysm is from bacterial endocarditis which was $80 \%$ in preantibiotic era. The source of aneurysm infection is septic embolization of microorganism from distant site or contagious spread of local infection. Most common pathogens are salmonella sp and staphylococcus aureus. ${ }^{1,2,3}$

Mitral stenosis is usually the result of recurrent rheumatic inflammation followed by healing. Mitral valvular disease is now found in parts of Asia and Africa predominantly in adolescents and young adult although occurred in western country in middle or old age. Most of the systemic arterial emboli lodges in cerebral arteries and can suddenly complicate or kill $10 \%$ of surgically untreated patients with mitral stenosis. In $25 \%$ of such patients, the emboli originated from left atrial thrombus with no residual thrombus in heart after embolization. Mitral valve replacement is the most common operation in mitral stenosis. Prosthetic valve endocarditis is an uncommon but serious complication that results death in more than half of the affected patients. Several series have reported rate of endocarditis of 0.06-04\%. ${ }^{4}$

Mycotic aneurysms have been reported in nearly every vessel including approximately $30 \%$ in visceral arteries, $30 \%$ in the aorta, and 3-4\% intracranially. As in the original case described by Osler, multiple mycotic aneurysms may be found in the same vessel. However, multiple mycotic aneurysms in different vessels in the same patient are rare. The bacteriology of these aneurysms is the same as that of infective endocarditis. Approximately $80 \%$ are Gram-positive cocci in origin; streptococci species comprise $60 \%$ and staphylococci species, $20 \% .126$ less than $13 \%$ are caused by Gram negative organisms. Ironically, less than $4 \%$ of mycotic aneurysms are caused by fungi. 1,5

Address of Correspondence: Dr. A.M. Asif Rahim , Department of Cardiac Surgery, National Institute of Cardiovascular Diseases, Dhaka, Bangladesh. e-mail-titormc@yahoo.com 
Clinically, abdominal pain, fever, bacteremia and pulsatile abdominal mass should suggest a mycotic aneurysm of abdominal aorta. This sort of abdominal aortic aneurysm has a tendency of early rupture especially more with infections due to salmonella. So mortality is very high in these rupture aneurysm in spite of emergency surgical management. ${ }^{3}$

Traditionally the treatment of mycotic aneurysm of aorta is excision of infected portion with proximal and distal closure, debridement of surrounding infected tissue, and extra-anatomic bypass (eg: axillo-bifemoral). Complication of proximal aortic stump (blowout) after this procedure, have led some to recommend in situ graft replacement after debridement of all infected tissue. ${ }^{1,3,5}$

\section{Case summary:}

A 11 years girl from Feni a school student of class IV admitted into national Institute of Cardiovascular diseases(NICVD) with the complains of pulsatile swelling in the abdominal wall that commenced with sudden lower abdominal pain two months ago. Initially it was diagnosed as nonspecific abdominal pain. Detailed investigation including CT angiogram was done. Previous CT angiogram revealed two saccular aneurysms in the distal part of superior mesenteric artery (SMA).Later on repeat CT angiogram done which revealed aneurysm in the distal part of aorta and also involving ostioproximal part of both the common iliac arteries as well as two saccular aneurysms in the distal part of SMA without features of rupture. She had a history of high fever and joint pain about 1 year back which was continuous, migratory in nature involving right knee, elbow and wrist joints. Thereafter she developed left sided hemi paresis ten months back diagnosed as right-sided intracranial subdural hematoma with acute hemorrhage producing mass effect. Right sided craniotomy with removal of heamatoma was done at neurosurgery department of Dhaka Medical College Hospital (DMCH).

With continued misfortune the girl subsequently developed severe mitral regurgitation with infective endocarditis. It was treated surgically at Cardiac surgery department of NICVD by systemic antibiotics followed by mitral valve replacement (MVR) with 27 $\mathrm{mm}$ Saint Judes bileaflet mechanical valve. Immediately after that MVR she experienced pain in her right lower limb from knee to foot associated with ulceration over knee joint and progressive blackening of lateral three toes. Her first CT angiogram was done that showed total occlusion of distal aorta just above the bifurcation including proximal two-third of both common iliac arteries. Patient underwent angioplasty of aortoilliac arteries with removal of clot by Fogerty embolectomy done at vascular surgery department of NICVD. These sequelae of MVR and Fogerty embolectomy were done each at 1 month interval following craniotomy in the same year. Drug history revealed that she took tab Phenoxymethyl Penicillin, tab Warfarin, Diuretics and other necessary treatment of each of three previous surgery.

On General examination ,patient was ill looking with average body build, mildly anaemic,pulse-84/ min ,regular, normal volume character, condition of vessel wall normal with no radio-femoral delay,BP-110/70 mm of $\mathrm{Hg}$, JVP-not raised, respiratory rate 18 breaths/min, scar mark in the skin over right knee and groin. Examination of the precordium revealed apex beat in left 5th intercostals space medical to midclavicular line normal with scar mark of median sternotomy incision of MVR surgery. Other system revealed no abnormality.

Local examination of right lower limb revealed scar mark of previous ulcer over right knee joint with residual weakness of both upper and left lower limb. All peripheral pulses were present normal in character and there were no muscle wasting.

Detail investigations including CT angiogram of abdominal aorta with distal runoff this time $\left(2^{\text {nd }}\right.$ time) showed fusiform dilatation in the distal part of Abdominal aorta(AA) including ostioproximal part of both common iliac arteries and saccular aneurysm in the distal part of Superior Mesenteric Artery. However CT angiogram 6 months back ( $1^{\text {st }}$ time prior to angioplasty and clot removal) revealed total occlusion of distal aorta just above the bifurcation also involving the proximal two-third of both common iliac arteries. Total long segment artery occlusion was noted in right SFA with mild narrowing of the ostioproximal part of hepatic artery. CT scan of brain this time revealed Post operative state showing right sided residual subdural haemorrhage and Right sided cerebral infarct with haemorrhagic transformation. Echocardiogram revealed post MVR status with good result. Other investigations for general assessment and anesthetic fitness were found with in normal range and limits. So final preoperative confirmed diagnosis was Mycotic aneurysms involving Abdominal Aorta, common iliac arteries and SMA.

This time patient underwent surgery at NICVD at vascular surgery department on 13.02.2011 


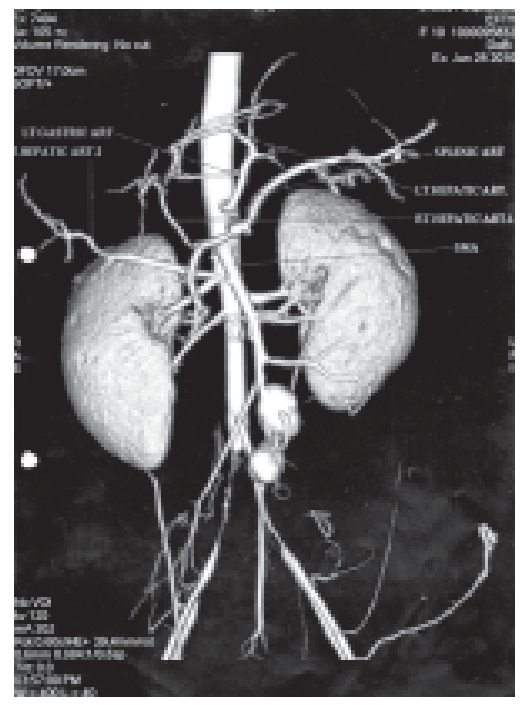

Fig.-1: $2^{\text {nd }}$ time $C T$ angiogram

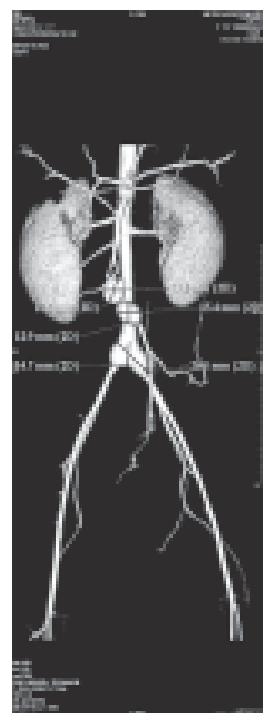

Fig.-2: $1^{\text {st }}$ time CT angiogram

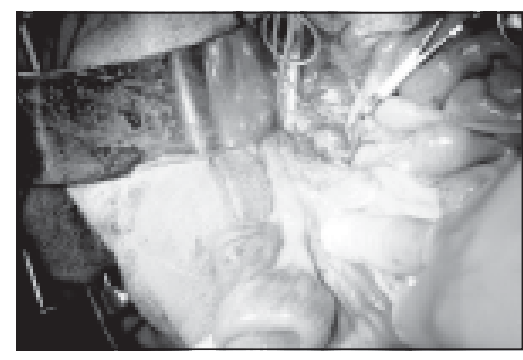

Fig.-3:

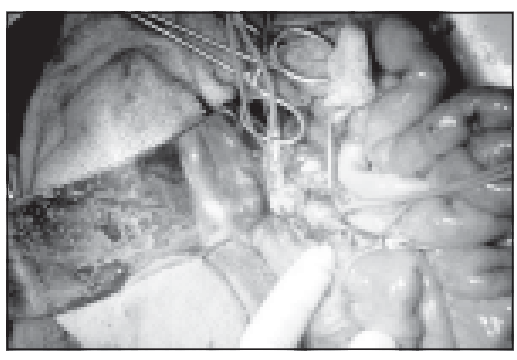

Fig.-4:

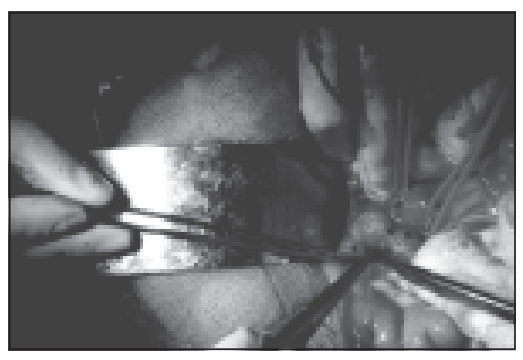

Fig.-5:

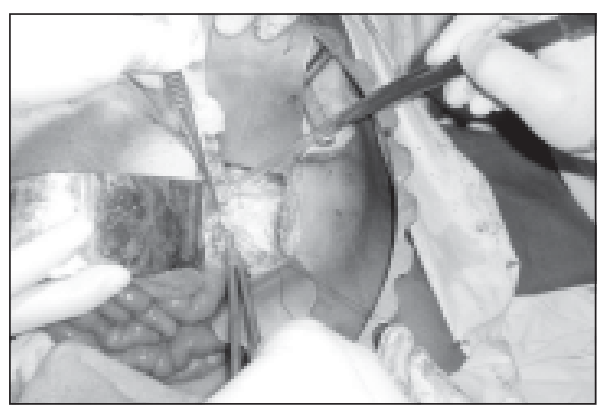

Fig.-6:

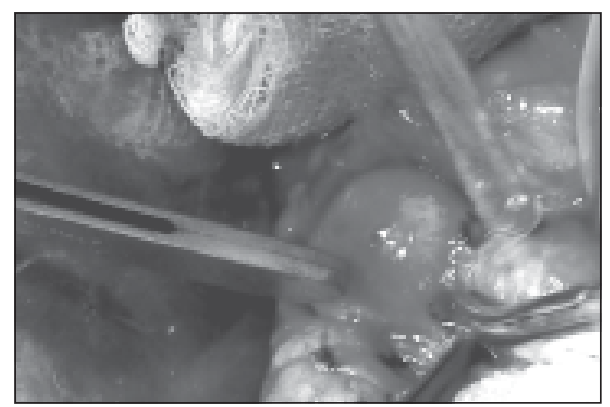

Fig.-7

Fig.-3 to 7: showing step of vascular surgery in mycotic aneurysm.

laparotomy done under G/A. Transperitoneally Mycotiv aneurysm in SMA and abdominal aorta(AA) were identified by manupullation of intestine and gut. After taping on SMA and AA, Aorto Bi-iliac bypass with PTFE (14/7mm)graft and Excision and ligation of SMA aneurysm were done and patient recovered uneventfully.

\section{Discussion:}

Mycotic aneurysm may occur in normal or atherosclerotic artery secondary to embolization of septic valvular vegetation in a patient of endocarditis or direct extension of endocardial origin, typically described as secondary and primary mycotic aneurysm. The most important 
factors in this aneurysm includes source of infection, nature of affected vessel and specific organism involved etc. Source of infection may be (i) intrinsic to vascular tree such as in the classic mycotic organism of Oslar, in which heavily colonized cardiac valve vegetations are the source of micro or macroemboli to distant sites. In these sites, the emboli lodge in vessel branch points or vasa vasorum accounting for the infection of large vessels. The infection may also spread contiguously and involve the adjacent ascending aorta. (ii)Extravascular or perivascular infection adjacent to aorta by means of bacterimia or by local spread may weaken the wall of blood vessel resulting in local aneurysm formation ( iii)Direct inoculation occurring from injection of illicit drugs, occasionally from invasive vascular procedures in the medical setting or from civilian or military trauma. Here in this case of postoperative phlebitis the central venous line, arterial line, or peripheral I/V channel may be one of the possible causes for mycotic aneurysm. As systemic sign symptoms of infection were not present like typical endocarditis related mycotic aneurysm, it could even occur due to simple recurrence following previous embolectomy.

The normal arterial wall is highly resistant to infection except in unusual circumstances. So infection can be promoted by local trauma due to some underlying disease or may be iatrogenic or accidental. A heavy innoculum of a very virulent organism is required to establish infection in a normal vessel wall. Although originally in late $19^{\text {th }}$ century the adjective "mycotic" referred to a spectrum of microorganism but yet recently the term is known to refer to fungi. Certain microorganism have long been known to have predilection for vascular infection, among these salmonella mostly involve aorta in $65 \%$ cases, staphylococcus common and streptococcus specially seen in endocarditis. Besides recently some bizarre infection like candida, aspergillus, compylobacter, clostridium, tuberculosis, histoplasma etc observed occasionally with vascular infection in patient with impaired immune status like diabetes, corticosteroid therapy, malnutrition. ${ }^{5,1}$

Most patients with mycotic aneurysm present with local and systemic sign and symptoms of infection like fever, leukocytosis, erythema, local tenderness etc. These usual sign symptoms were not present in this patient except vague abdominal pain, swelling in abdominal wall. The danger of mycotic aneurysm especially in great vessel like aorta is impending rupture that threatens the life of the patient. CT angiogram revealed saccular aneurysm with narrow neck giving lobulated appearance. ${ }^{5}$

Surgical treatment of mycotic aneurysm is always site specific. Aneurysm in nonessential noncritical artery should be managed by proximal and distal ligation from the site of infection with excision of infected segment that was done here in this patient in SMA mycotic aneurysm. In this process even femoral artery can be sacrificed but more frequently it requires bypass. So the challenge for vascular surgeon in situations where infected artery must be replaced or bypassed is to provide adequate circulation for essential organs or limbs. In-line grafting or endoaneurysmorraphy can also be done alternatively. ${ }^{5}$

Here as mycotic aneurysm involves abdominal aorta with both common iliac arteries and SMA in this case so it can be easily termed a type aortoiliac occlusive disease. Aortoilliac Endarterectomy, Aortobifemoral bypass graft, illiofemoral graft and other indirect methods of revascularization eg. Axillobifemoral bypass are the main surgical treatment option. Angioplasty of aortoilliac arteries with removal of clot by Fogerty embolectomy done first time in this case is debatable. Aorto Bi-iliac bypass was not done as patient was very young, to avoid possible high chance of graft related complications and increased risk of infection with nonautogenous reconstruction. Second time we rightly did Aorta Bi-iliac bypass with PTFE $(14 / 7 \mathrm{~mm})$ graft and Excision and ligation of SMA aneurysm, that has excellent outcome generally. 85-90\% graft remains patent for 5 years and $70-75 \%$ for 10 years with perioperative mortality around $1-2 \%$. In broad heading complications of aortoilliac surgery with graft replacement include infection $0.5-3 \%$, haemorrage, thrombosis 1-2\%, embolization 2-5\%, ischemic colitis $0.5-6 \%$, rarely paraplegia, and pseudoaneurysm in $3-15 \%$ cases. $10-20 \%$ of patients may experience occlusion of a graft limb and anastomotic aneurysm of aortic graft insertion 3$5 \%$. Avoidance of infection of the autogenous or 
synthetic reconstruction is often difficult and may require ingenuity. ${ }^{3,5}$

\section{Conclusion:}

Mycotic aneurysm is not very common in our situation here at National Institute of Cardiovascular Diseases following MVR Wholehearted precaution, strict asepsis, sincerity, skill are necessary to prevent development of this sort of mycotic aneurysm and its recurrence in patients with valve surgery. With appropriate treatment, both medical and surgical, this sort of complications can be managed.

\section{References:}

1. Parler BA, Ernst CB. Infected Aneurysms.In: Robert W, Hobson II, Samuel E Wilson, Frank J.Veith. Eds.
Vascular surgery Principles and Practice. New York: Marcel Dekkar Inc, 1994: 669-693.

2. Jimenez JC, Wilson SE. Aneurysmal disease.In: Rodney A White, Larry H Hollier. Eds. Vascular Surgery: Basic Science and Clinical. UK: Blackwell Publishing, 2005:162-179

3. Schermerhorn ML, Cronenwett JL. Abdominal Aortic and Iliac Aneurysms. In: Rutherford Vascular surgery. UK: Elsevier, 2005:1408-1452

4. Kouchoukos NT, Robert B,Frank L.Mitral Valve Disease with or without Tricuspid valve Disease. In: Kirklin / Barratt Boyes . Eds. Cardiac Surgery. USA: Churchill Livingstone, 2003: 483-553

5. McCann RL, Sebastian MC. Mycotic Aneurysms. In: Richard H Dean, James S.T.Yao, David C.Brewster. Eds. Current Diagnosis \& treatment in Vascular Surgery. USA: Prentice-Hall International Inc.1995:253-262 